\title{
Mycobacterium tuberculosis causing tuberculous lymphadenitis in Maputo, Mozambique
}

Sofia Omar Viegas ${ }^{1,2,3^{*}}$, Solomon Ghebremichael ${ }^{4}$, Leguesse Massawo ${ }^{1}$, Matos Alberto ${ }^{5}$, Fabíola Couto Fernandes ${ }^{5,6}$, Eliane Monteiro ${ }^{5}$, David Couvin ${ }^{7}$, José Maiane Matavele ${ }^{1}$, Nalin Rastogi ${ }^{7}$, Margarida Correia-Neves ${ }^{3,8,9}$,

Adelina Machado², Carla Carrilho5,6, Ramona Groenheit ${ }^{4}$, Gunilla Källenius ${ }^{3}$ and Tuija Koivula $a^{3,4}$

\begin{abstract}
Background: The zoonosis bovine tuberculosis (TB) is known to be responsible for a considerable proportion of extrapulmonary TB. In Mozambique, bovine TB is a recognised problem in cattle, but little has been done to evaluate how Mycobacterium bovis has contributed to human TB. We here explore the public health risk for bovine TB in Maputo, by characterizing the isolates from tuberculous lymphadenitis (TBLN) cases, a common manifestation of bovine TB in humans, in the Pathology Service of Maputo Central Hospital, in Mozambique, during one year.

Results: Among 110 patients suspected of having TBLN, 49 had a positive culture result. Of those, 48 (98\%) were positive for Mycobacterium tuberculosis complex and one for nontuberculous mycobacteria. Of the 45 isolates analysed by spoligotyping and Mycobacterial Interspersed Repetitive Unit - Variable Number Tandem Repeat (MIRU-VNTR), all were M. tuberculosis. No M. bovis was found.

Cervical TBLN, corresponding to 39 (86.7 \%) cases, was the main cause of TBLN and $66.7 \%$ of those where from HIV positive patients.

We found that TBLN in Maputo was caused by a variety of $M$. tuberculosis strains. The most prevalent lineage was the EAI ( $n=19 ; 43.2 \%$ ). Particular common spoligotypes were SIT 48 (EAl1_SOM sublineage), SIT 42 (LAM 9), SIT 1 (Beijing) and SIT53 (T1), similar to findings among pulmonary cases.

Conclusions: M. tuberculosis was the main etiological agent of TBLN in Maputo. M. tuberculosis genotypes were similar to the ones causing pulmonary TB, suggesting that in Maputo, cases of TBLN arise from the same source as pulmonary $T B$, rather than from an external zoonotic source. Further research is needed on other forms of extrapulmonary TB and in rural areas where there is high prevalence of bovine TB in cattle, to evaluate the risk of transmission of M. bovis from cattle to humans.
\end{abstract}

\section{Background}

Tuberculosis (TB) ranks as the second leading cause of death from a single infectious agent, after the human immunodeficiency virus (HIV) [1]. In 2013, an estimated 9 million people developed TB and 1.5 million died from the disease, from whom 360000 were HIV positive [1]. The African continent accounts for one quarter of all $\mathrm{TB}$ cases in the world and also has the highest rates of cases and deaths relative to population [1].

\footnotetext{
*Correspondence: viegas_sofia@hotmail.com

${ }^{1}$ National Institute of Health, Ministry of Health, Maputo, Mozambique

${ }^{2}$ Faculty of Veterinary, Eduardo Mondlane University, Maputo, Mozambique

Full list of author information is available at the end of the article
}

Mozambique is one of the high burden TB and HIV countries with a prevalence of HIV infection in adults of $11.5 \%$ [2] and an estimated TB prevalence of 559 per 100000 population. Fifty six percent of the TB patients in Mozambique are estimated to be HIV positive [1]. Among all reported TB cases in 2013, 9.8 \% were extrapulmonary [1].

TB is caused by bacteria of the Mycobacterium tuberculosis complex. The Mycobacterium tuberculosis and the Mycobacterium bovis are the primary agents of the disease in humans and cattle respectively.

The TB epidemic in Mozambique is caused by an extensive diversity of $M$. tuberculosis spoligotypes with 
predominance of LAM, EAI, T and Beijing lineages [3]. To our knowledge, no information regarding lineages involved in extrapulmonary TB in Mozambique is available.

Bovine TB, caused by M. bovis, is the main zoonotic disease caused by mycobacteria, affecting cattle, other domesticated animals and several free or captive wildlife species. In Mozambiquethe overall prevalence of BTB in cattle is $13.6 \%$ [4], varying from $0.98 \%$ in Massingir [5] to $39.6 \%$ in the Govuro district [6]. Little is known about the impact of bovine $\mathrm{TB}$ as a human disease in low income countries, particularly in HIV positive patients, including in Mozambique.

In Africa, bovine TB accounts for an estimated median proportion of $2.8 \%$ (range $0 \%-37.7 \%$ ) of all reported human TB cases [7].

Bovine TB is spread to humans, typically by ingestion of unpasteurized milk or contaminated meat, causing extrapulmonary $\mathrm{TB}$, but can also be transmitted by inhalation of aerosols causing pulmonary TB $[8,9]$. Several studies have detected $M$. bovis in tuberculous lymphadenitis (TBLN) cases [10-15], being the most common among all extrapulmonary TB cases.

In the present study, we explored the public health risk for bovine TB in Maputo, capital of Mozambique, by characterizing the isolates from TBLN cases, during one year, in the Pathology Service of Maputo Central Hospital.

\section{Results}

\section{Demographic characteristics}

Participants were recruited from July 2013 to July 2014 at the Pathology Service of Maputo Central Hospital, Mozambique. A total of 110 patients, suspected of having TBLN, were recruited to participate in the study (Fig. 1). From those patients, 45 isolates were analysed by genotyping methods, one isolate per patient. The demographic information of the 45 patients is summarized in Table 1. Among all, 28 (62.2 \%) were male while 17 (37.8 \%) were female.

The patients' median age was 33 years (11.1 SD) with a range of 18-75 years. Stratification according to age showed that $39(86.7 \%)$ of the patients were aged 18-45, while $6(13.3 \%)$ were above 46 years.

Fifteen (33.3\%) patients had been previously treated for TB and 15 (33.3\%) had had previous contact with TB patients.

\section{Site of sample collection}

Cervical lymphadenitis was the main cause of TBLN in Maputo. Of the lymph node samples 39 (86.7\%) were collected from the cervical region, two (4.4\%) from axillary site and one (2.2\%) from inguinal region. Other sites were breast, chest and thigh (one case, $2.2 \%$ from each site).

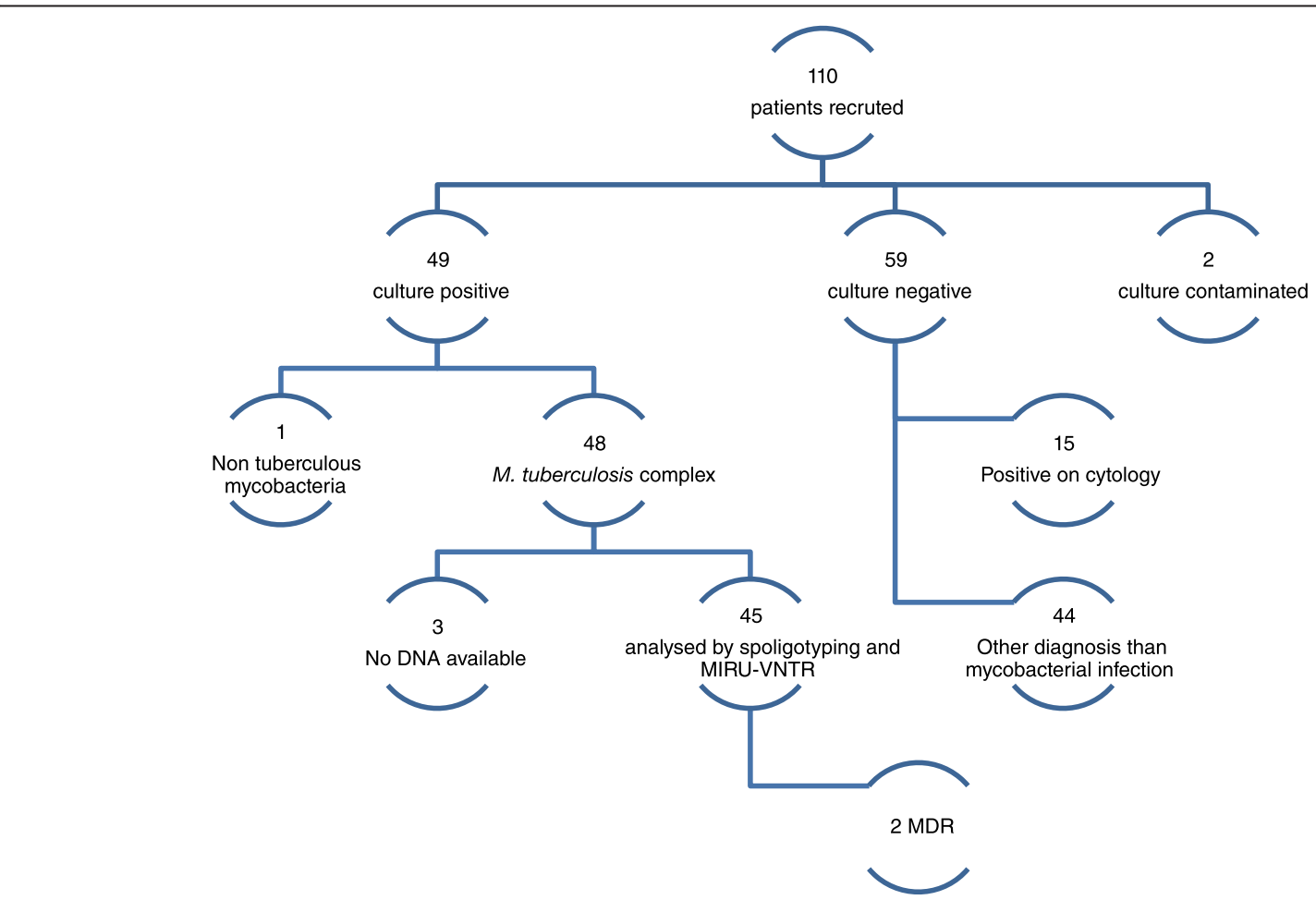

Fig. 1 Chart showing patient's recruitment, culture, identification and resistance results 
Table 1 Demographic data of the 45 patients that were analysed by genotyping methods

\begin{tabular}{llc}
\hline Category & Total isolates & $\mathrm{n}(\%)$ \\
\hline Sex & Male & $28(62.2)$ \\
Age & Female & $17(37.8)$ \\
& $18-45$ & $39(86.7)$ \\
HIV sero-status & +45 & $6(13.3)$ \\
& Positive & $30(66.7)$ \\
& Negative & $9(20)$ \\
Previously TB treatment & Not tested for HIV & $6(13.3)$ \\
& Yes & $15(33.3)$ \\
Contact with TB patients & No & $30(66.7)$ \\
& Yes & $15(33.3)$ \\
& No & $30(66.7)$ \\
\hline
\end{tabular}

\section{HIV serology and drug resistance}

Among the 45 patients, 30 (66.7\%) were HIV positive (19 males and 11 females), nine (20.0 \%) were HIV negative and six (13.3\%) were not tested for HIV. Of the cervical TBLN cases, 26 (66.7\%) were HIV positive patients. Of the 61 patients with negative or contaminated culture results, 39 (63.9 \%) were HIV positive, nine (14.8\%) were HIV negative and 13 (21.3 \%) were not tested for HIV. No statistical association was found between HIV serology and cervical TBLN.

In Mozambique, being a mine worker is considered a risk factor for HIV [16], information related to previous work in South African mines was collected; among all positive cultures, four were from mine workers, of them two were HIV positive and two HIV negative.

Two patients, EBOV 13-23 (27 years, Lineage T1, SIT 53) and EBOV 13-29 (28 years, Lineage Beijing, SIT 1 ), both males, HIV positive and not previously treated for $\mathrm{TB}$, were diagnosed with multidrug resistant (MDR) TB, defined as simultaneous resistance to isoniazid and rifampicin (Fig. 2).

\section{Culture and cytology results}

Among all, 49 patients had a positive mycobacterial culture, giving a culture positivity rate of $44.5 \%$. Of them, 48 isolates were identified as $M$. tuberculosis complex and one as nontuberculous mycobacteria (NTM; 32 years, male, HIV positive). From the 59 culture negative patients there were an additional 15 (25.4\%) cases Ziehl Neelsen

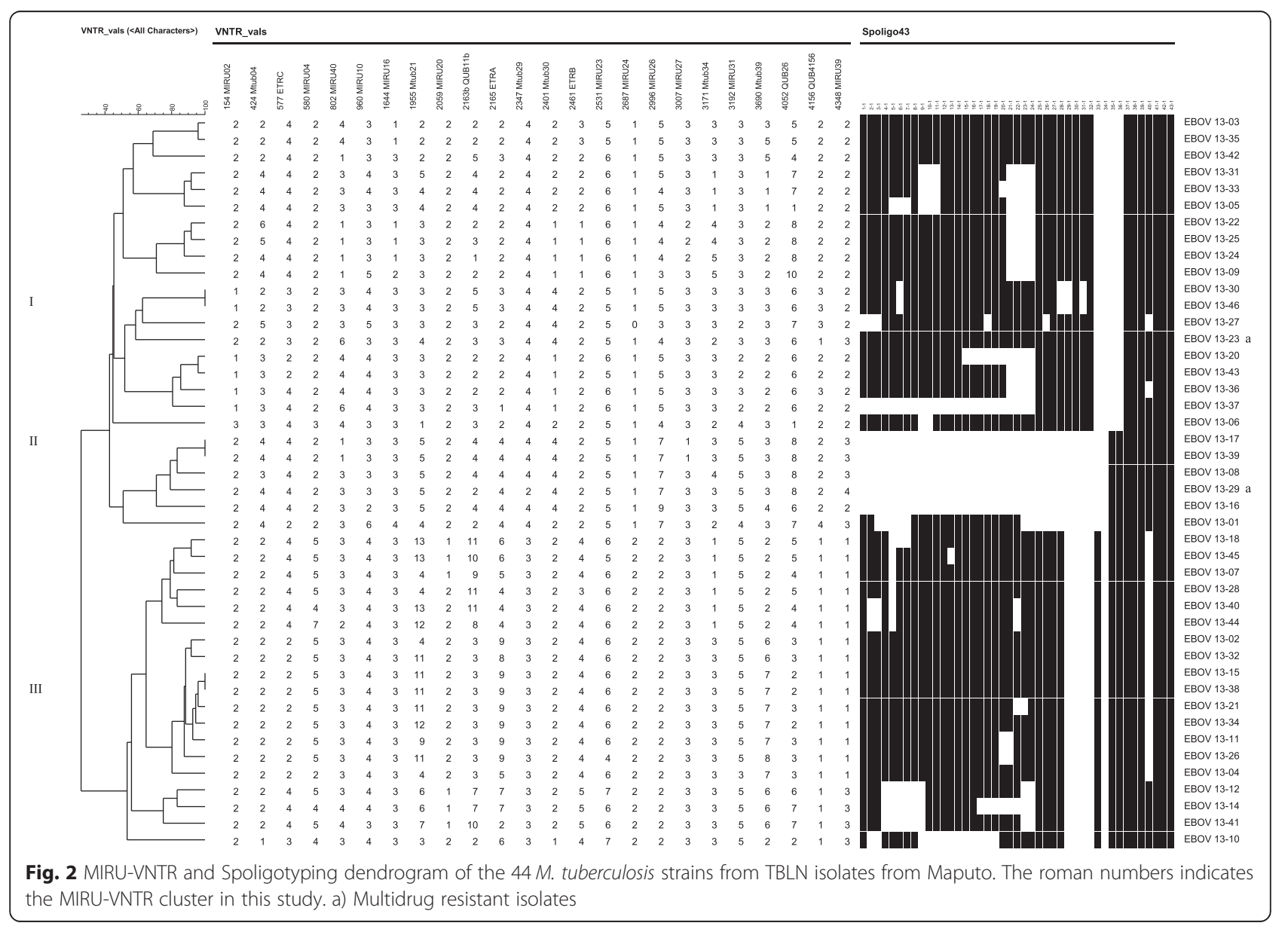


(ZN) positive on cytology (morphological evidence of mycobacterial infection). In the remaining 44 patients, based on cytology, there was a specific diagnosis other than mycobacterial infection.

Among the 48 culture confirmed TB cases, 45 isolates were analysed by spoligotyping and Mycobacterial Interspersed Repetitive Unit-Variable Number Tandem Repeat (MIRU-VNTR). For the remaining three isolates and for the NTM, DNA was not available, because there was no growth during the re-culture procedure (Fig. 1).

\section{Mixed infection isolate}

In patient EBOV 13-19 (male, 59 years, HIV positive), a spoligotype SIT 2117 (Manu 2; all spacers present except spacers 9, 10, 33 and 43) was observed (Additional file $1)$. This type of pattern may correspond to a mixed infection, due to concomitant Beijing and Euro-American lineage strains (the latter comprising $\mathrm{H}, \mathrm{LAM}, \mathrm{X}$, and $\mathrm{T}$ lineages per spoligotyping defined clades). A mixed infection was defined as the occurrence of strains with different 24-loci MIRU-VNTR patterns at two or more loci in the same sample. We further investigated this isolate; by spoligotyping we could observe different intensities of the spacers in the spoligotyping pattern (Fig. 3) and by MIRU-VNTR we could observe double alleles in three different locus (MIRU26, Mtub21, and Mtub30), confirming a mixed infection pattern. Since it was confirmed as a mixed infection, the isolate EBOV 13-19 was excluded from further genotypic analysis.

\section{Spoligotyping}

Spoligotyping is a simple, rapid and cost effective method for simultaneous detection and typing of the $M$. tuberculosis complex. It is the method of choice for strains with less than five copies of the insertion sequence IS6110, like M. bovis strains, which usually contain only one or two IS6110 copies [17-19]. By spoligotyping, $M$. tuberculosis isolates are characterized by the absence of spacers 33-36, while $M$. bovis usually lack spacers 39-43 [20].

Spoligotyping was performed on 45 isolates. Of them, all were defined as $M$. tuberculosis and no $M$. bovis was found.

Among the 44 isolates (excluding the mixed infection isolate), 23 spoligopatterns were obtained. Three patterns corresponded to orphan strains that were unique in the SITVIT2 database, as opposed to 20 patterns from 41 patients that corresponded to shared-types (SITs), i.e. an identical pattern shared by two or more patients worldwide (within this study, or matching another strain in the SITVIT2 database), as shown in Table 2.

For each isolate, their binary/octal description, their lineages and SITs are summarized in Table 2. Four SITs (containing seven isolates) were newly created either within the present study or after a match with an orphan in the database. Nine patterns were in clusters, containing 30 isolates ( 2 to 6 isolates per cluster), amounting to an overall clustering rate of $68.2 \%(30 / 44)$.

As shown in Table 2, the most common spoligotypes found in this study were SIT48 (East African-Indian_ Somalia; EAI1_SOM) with six isolates; SIT1 (Beijing lineage) and SIT42 (Latin America Mediterranean; LAM 9) with five isolates each and SIT53 (T1) with four isolates. The most common lineage was the EAI $(n=19 ; 43.2 \%)$.

\section{MIRU-VNTR results}

Figure 2 shows the MIRU-VNTR clusters and respective spoligotyping results obtained in this study. Among the 44 isolates analysed by MIRU-VNTR, a wide variety of patterns were observed. Only three clusters of two isolates each were formed, cluster I (Lineage H3, SIT 4094); cluster II (Beijing lineage, SIT 1); cluster III (Lineage EAI1_SOM, SIT 48). The remaining patterns were unique, i.e., did not cluster with any other isolate within this study.

\section{Phylogenetic analysis}

Minimum Spanning Trees (MST) depicts evolutionary relationships between the $M$. tuberculosis genotypes in our study using spoligotyping and/or 24-loci MIRUVNTR typing. The MST based on spoligotypes alone (Additional file 2A) showed isolated relatively well clustered into their respective lineages/sublineages. Isolates belonging to Beijing, EAI lineage and LAM were rather well organized in the three MSTs. However, on the MST based on 24-loci MIRU-VNTR alone (Additional file 2B), one may notice that the isolate represented by SIT254 or 24-MIT Or16 (lineage T5-RUS1), was better correlated with isolates belonging to LAM lineage. Furthermore, the isolate represented by SIT4 or 24-MIT Or30 (Unknown lineage) was also close to LAM lineage strains. Contrary to the MST based on spoligotypes alone (Additional file 2A) and on spoligotypes + 24-loci MIRU-VNTR (Additional file 2C), the MST based on 24-loci MIRUVNTR alone (Additional file 2B) depicted a similarity between isolates belonging to Beijing lineage and the unique isolate (SIT952 or 24-MIT Or01) representing CAS1-

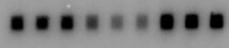

Fig. 3 Spoligotyping pattern of the strain EBOV 13-19. The picture shows different intensities of the spacers 
Table 2 Shared Types and orphans description in the study, their percentage and lineage within the study

\begin{tabular}{|c|c|c|c|c|c|}
\hline SIT* & Spoligotype Description & Octal code & Total in this study & $\%$ in this study & Lineage \\
\hline 1 & 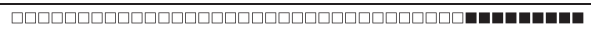 & 000000000003771 & 5 & 11.36 & Beijing \\
\hline 42 & 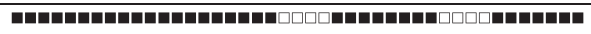 & 777777607760771 & 5 & 11.36 & LAM9 \\
\hline 48 & " & 777777777413731 & 6 & 13.64 & EAl1-SOM \\
\hline 53 & | & 777777777760771 & 4 & 9.09 & T1 \\
\hline 129 & " & 700777747413771 & 2 & 4.55 & EAI6-BGD1 \\
\hline 806 & " & 757777777413731 & 2 & 4.55 & EAI1-SOM \\
\hline 4 & | & 000000007760771 & 1 & 2.27 & Unknown \\
\hline 33 & " & 776177607760771 & 1 & 2.27 & LAM3 \\
\hline 34 & 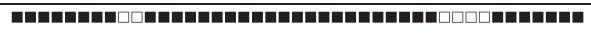 & 776377777760771 & 1 & 2.27 & $\mathrm{~s}$ \\
\hline 60 & 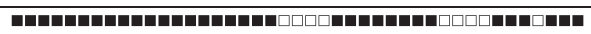 & 777777607760731 & 1 & 2.27 & LAM4 \\
\hline 254 & 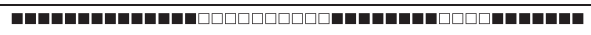 & 777760007760771 & 1 & 2.27 & T5-RUS1 \\
\hline 719 & " & 776177407760771 & 1 & 2.27 & LAM3 \\
\hline 952 & 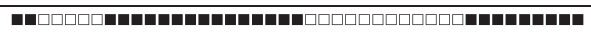 & 603777740003771 & 1 & 2.27 & CAS1-Delhi \\
\hline 1062 & 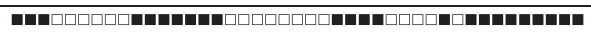 & 700774007413771 & 1 & 2.27 & EAI5 \\
\hline 2022 & 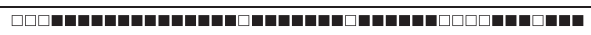 & 077776775760731 & 1 & 2.27 & $\mathrm{X} 1$ \\
\hline 2915 & 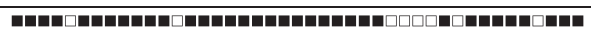 & 757737777413731 & 1 & 2.27 & EAI1-SOM \\
\hline $4092^{*}$ & 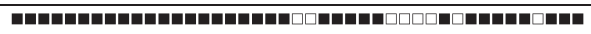 & 777777717413731 & 1 & 2.27 & EAl1-SOM \\
\hline $4093^{*}$ & " & 457777737413731 & 2 & 4.55 & EAl1-SOM \\
\hline $4094^{*}$ & 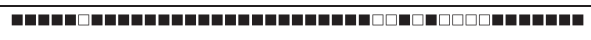 & 767777777120771 & 2 & 4.55 & $\mathrm{H} 3$ \\
\hline $4095^{*}$ & " & 777777477413731 & 2 & 4.55 & EAl1-SOM \\
\hline Orphan $^{\star *}$ & (1) & 476000377413771 & 1 & 2.27 & EAI5 \\
\hline Orphan $^{\star *}$ & 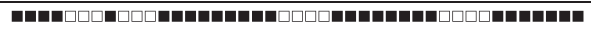 & 742177607760771 & 1 & 2.27 & LAM9 \\
\hline Orphan $^{\star *}$ & 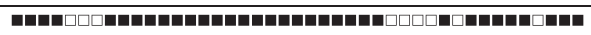 & 743777777413731 & 1 & 2.27 & EAl1-SOM \\
\hline
\end{tabular}

*Newly created SITs, either within the present study or after a match with an orphan in the database **Orphans, unique in the SITVIT2 database

Delhi lineage. For a better data mining of isolates related to 24-loci MIRU-VNTR information, one can refer to Additional file 1 showing the whole picture of association between the various genotypes.

\section{Discussion}

In this study we presented for the first time the lineages of $M$. tuberculosis complex causing extrapulmonary TB in Maputo, Mozambique. Extrapulmonary TB is reported in $11.6 \%$ of all TB cases in the country [1], the majority of them being TBLN.

Several studies have shown correlation between HIV infection and TBLN [21-23]. The synergies between TB and HIV infection [24, 25] have resulted in an increase in the incidence of TBLN and have further complicated
TB control. In this study, the high prevalence of HIV positive patients $(66.7 \%)$ among TBLN cases, might suggest a rising trend of HIV infection associated with TBLN in Maputo. A possible explanation for the high rate of HIV among TBLN cases might be confounders associated with HIV. However, the numbers related to previous mine working did not allow the interpretation of potential associations with HIV and information related to alcohol, drug abuse, ex-imprisonment or smoking was not collected.

Laboratory detection of bovine TB is a challenge, particularly in low income countries. Microscopy for mycobacteria on the FNA is the initial diagnostic procedure for lymphadenitis in Mozambique; although it does not differentiate between M. tuberculosis and M. bovis, it is 
considered a reliable TBLN diagnostic method, including in HIV positive individuals [26-29]. Molecular typing methods for $M$. tuberculosis complex detection on FNA specimen are costly and require technical expertise, therefore, are not implemented as a routine method in the country, making the detection of bovine TB difficult.

In this study, among all TBLN suspects, $43.6 \%$ were confirmed to have M. tuberculosis complex strains on culture and one was NTM, no M. bovis was found, showing that $M$. tuberculosis is the main cause of TBLN in Maputo. The additional 15 (25.4\%) cases that were positive on cytology might be due to infection by either M. tuberculosis complex or NTM which were not detected by culture.

These results are compatible with two studies conducted in the North of Ethiopia, where no M. bovis was detected and $M$. tuberculosis was identified as the main etiological agent in TBLN cases [30,31]. On the other hand, another study conducted in Guji zone of Ethiopia, an area inhabited by pastoral and agro-pastoral communities whose livelihood is based on livestock production, among 173 isolates, three were $M$. bovis; the same study analysed 39 livestock samples, where one $M$. tuberculosis was isolated in camels, suggesting transmission between livestock and humans in this pastoral area. This last study emphasises the importance of an appropriate study area, where risk factors, including close contact between humans and livestock, and consumption of raw milk and meat by the communities, are present. This can be one of the reasons for the absence of $M$. bovis in the present study.

By spoligotyping, the main lineages of TBLN were the EAI, Beijing; LAM and T1; and the major SITs, were SIT48, SIT1, SIT42 and SIT53. These genotypes are also predominant in pulmonary cases in Mozambique [3], indicating that there are no differences in the population of strains in pulmonary and extrapulmonary cases. Similarities between pulmonary and extrapulmonary cases were observed in other countries, i.e. Ethiopia [31], Thailand [32], Madagascar [33] and Brasil [34].

In African countries, little is known about the common lineages responsible for extrapulmonary TB. A study conducted in the neighbouring South Africa, in children, showed similarities with our findings, stressing the proximity between the two countries. In the South African study, $21.2 \%$ of the M. tuberculosis isolates belonged to the LAM lineage, and $20 \%$ to the Beijing lineages [35]. In another study performed in Ethiopia, spoligotyping revealed that the most common spoligotypes in extrapulmonary TB were SIT54, SIT53, and SIT149 [36]; SIT54, Lineage T1 was also found to be common in TBLN cases from the present study.

The ancestral EAI lineage, most predominant in this study (42.2\%), and one of the prevalent lineages in pulmonary TB cases in Mozambique, with a predominance of $29.7 \%$ [3]; is considered to be endemic in the Southern region of India [37, 38]. The migration link between India, particularly South India, and Mozambique arisen since the second half of the 19th century, when Indian traders practised the trade routes of the Indian Ocean, for transnational connections. The high prevalence of EAI lineages in Maputo might represent an indication of TB transmission between the two countries.

The Beijing lineage was also found to be one of the most common lineages in TBLN cases from this study. In South Africa, it was the second most common spoligotype found in extrapulmonary cases [35] and in Thailand, Beijing lineage was reported to be the most predominant in extrapulmonary TB cases, with 56 [39] and $57.9 \%$ [40]. Further research is needed to evaluate whether Beijing lineage has any particular association with extrapulmonary TB.

We have also shown an association between the Beijing lineage and HIV infection [41], although in this study, perhaps because of the sample size, it was not possible to find any relationship between a particular lineage and HIV infection. Furthermore, analysis of the spoligotyping lineages did not show any association with a particular clinical expression of the disease (data not shown).

Based on MIRU-VNTR analysis, we could observe a wide diversity of patterns, within strain lineages, showing that TB lymphadenitis in Maputo is not caused by a particular strain but by a wide variety of strains, an indication that risk factors for developing TBLN are rather associated with host than $M$. tuberculosis strain.

In this study, a mixed $M$. tuberculosis complex infection was detected in one case of TBLN. Mixed M. tuberculosis infections, a potential obstacle for tuberculosis treatment and control, occurs when an individual is simultaneously infected with more than one strain of $M$. tuberculosis complex. In high $\mathrm{TB}$ prevalence settings, mixed infections are frequent, implying high reinfection rates and the absence of efficient protective immunity conferred by the initial infection [42].

In pulmonary $\mathrm{TB}$ cases from Mozambique, we have shown mixed infection with a Beijing and non-Beijing strain in two out of five Manu strains [3].

In South Africa, a study conducted in Cape Town, showed that $57 \%$ of patients infected with a Beijing strain were also infected with a non-Beijing strain [42]. Other countries have also reported mixed infection within different strains from the $M$. tuberculosis complex, i.e. Botswana [43], China [44], Taiwan [45].

In this study, no $M$. bovis was found. In low income countries there are no effective animal TB control programmes and surveillance, and the epidemiological and public health aspects of infection due to bovine TB are scarce [7-9]. This situation is aggravated by the presence 
of additional risk factors such as human behaviour and the high prevalence of HIV infections [8, 9, 13]. In African countries, a median of $2.8 \%$ (range $0 \%-37.7 \%$ ) of all humans cases of TB are estimated to be caused by $M$. bovis [7]. Variances on prevalence are observed in different sites; those differences might be influenced by sampling, study area and diagnostic methods. Prevalence varies from; 17 and $4.4 \%$ in Ethiopia [12, 46], 16 in Tanzania [47], 7 in Uganda [14], 3 in Ghana [48] and $15.38 \%$ in Nigeria [49]. The genotyping findings from this study and the findings in pulmonary cases [3] indicate that the overall contribution of $M$. bovis to human TB in Maputo is minor. However, the present study has certain limitations. The small number of positive cases on culture might have reduced the chances of finding M. bovis as well as the statistical power and have affected the conclusions regarding the significance of the different variables and $M$. tuberculosis lineages. Furthermore, patients from this study are from urban or peri-urban areas of Maputo where livestock and consumption of unpasteurized milk is minor, thus, exposure to $M$. bovis is less.

The occurrence of zoonotic TB is greatly dependent on the presence of bovine TB in cattle. $M$ bovis in cattle is very frequent in certain areas of Mozambique; a recent publication has demonstrated a high prevalence of bovine TB in cattle of $39.6 \%$ (95 \% CI 36.8-42.5) in one particular district of Mozambique [6]. Another study carried out in 2008 in the same region reported a bovine TB prevalence rate of $61.9 \%$ (95\% CI: 55.8-67.8) [50]. Further research is needed on cases of abdominal TB and other forms of $\mathrm{TB}$, and in pastoral areas, where the prevalence of bovine TB in cattle is known to be high in order to have a better answer about the public health importance of this zoonotic disease in Mozambique.

\section{Conclusions}

M. tuberculosis was the main etiological agent of TBLN in Maputo. M. tuberculosis lineages in TBLN were similar to the ones previously reported to cause pulmonary TB, suggesting that in Maputo, cases of TBLN arise from the same source as pulmonary $\mathrm{TB}$, rather than from an external zoonotic source.

\section{Methods}

\section{Ethical considerations}

Institutional permission to conduct the study was obtained from the National Bioethics Committee of the Ministry of Health in Maputo, Mozambique, reference number 216/ CNBS/14. The patients were included after understanding the study and had signed an informed consent.

\section{Study setting}

In Mozambique there are three pathology services, in Maputo, Beira and Nampula, each of them responding for the South, Central and North region of the country respectively.

The Pathology Service of Maputo Central Hospital is the only referral site in Maputo for diagnosing TBLN, patients suspected of mycobacterial infection were referred from different health units for diagnosis. During the study period, they have received 677 patients suspected of TBLN, of whom, 110 (16.2\%) were included in the study. The swellings observed were cervical, axillary or from other sites, either as a unilateral single or multiple mass or masses. Fistula formation could also been seen in certain cases.

Patients who also had pulmonary involvement were considered as extrapulmonary TB in our analysis.

\section{Patients and specimens}

This study was conducted from July 2013 to July 2014 at the Pathology Service of Maputo Central Hospital in Maputo, Mozambique. A total of 110 patients with suspected TBLN and subjected to FNA were included in the study. For each patient, a questionnaire was applied.

Demographic and clinical information of the participants was collected by trained clinical nurses using a pre-tested questionnaire. HIV test results were collected from the medical records after obtaining written consent from the patients. Patients without HIV results were advised for testing and that was only performed after patients consent.

Only patients suspected of TBLN that consented and could give at least $0,1 \mathrm{ml}$ of sample were recruited to participate in the study.. That was applied in order to have enough material for the routine smear performed in the unit, direct microscopy using conventional $\mathrm{ZN}$ staining and cytology, and subsequent assays to be performed within the study.

All material was collected by a Specialist Physician from the Pathology Service, using the FNA Cytology procedure in use in the Unit, as described below.

\section{Collection of lymph node aspirate and staining}

Lymph node aspirate was collected as a routine procedure in the Pathology Service for all suspected of having TBLN. Briefly FNA was performed from a swollen superficial lymph node by using a sterile 23-gauge needle with an attached $10 \mathrm{ml}$ syringe. The overlying area was cleaned with $70 \%$ alcohol. Then the node was punctured by developing a negative pressure in the syringe. Multiple in and out passes were made by the needle without exiting the node. After removing the needle, in all cases, special $\mathrm{ZN}$ staining for acid fast bacilli was done and cytology was performed. The remaining aspirated sample was referred to The National TB Reference Laboratory for culture. 


\section{Culture and identification methods}

The cultures were performed using N-Acetyl-L-Cysteine-Sodium Hydroxide (NALC-4 \% $\mathrm{NaOH}$ ) decontamination method as previously described [51] and inoculated into three culture media: 1) Liquid culture (BD $\mathrm{BBL}^{\mathrm{mm}}$ MGIT $^{\text {ma }}$ Mycobacteria Growth Indicator Tube), 2) Löwenstein-Jensen slants (BD BBL ${ }^{\text {Tux }}$ Lowenstein-Jensen Medium) and 3) Stonebrinkslants (in house made-). All tubes were incubated at $37{ }^{\circ} \mathrm{C}$.

All positive cultures were accordingly identified as $M$. $t u$ berculosis complex or not using SD BIOLINE TB Ag MPT64 rapid test according to manufacturer's instructions.

Isolates identified as $M$. tuberculosis complex were subjected to Line Probe Assay, GenoType MTBDRplus (Hain, Nehren, Germany), for detection of MDR TB as previously described [52].

\section{Spoligotyping}

Standard spoligotyping [53] was performed generally as described by Kamerbeek and colleagues. Spoligotyping results were analysed and dendograms created using the BioNumerics Software ver. 7.5 (Applied Maths, Kortrijk, Belgium).

Spoligotyping patterns were also compared with the ones existing in the international Spoligotyping database SITVIT2, which is an updated version of SITVITWEB [54]. Shared international types (SITs) that were newly-created either within the present study or after a match with an orphan in the database, were assigned a new SIT number.

\section{Mycobacterial Interspersed Repetitive Unit - Variable Number Tandem Repeat (MIRU-VNTR) analysis}

Standardized 24-loci MIRU-VNTR typing [55] was performed using the MIRU-VNTR typing kit (Genoscreen, Lille, France). The PCR-products were run with 1200 LIZ size standard (GeneScan, Applied Biosystems) on ABI3500 sequencers. Sizing of the PCR-fragments and assignments of MIRU-VNTR alleles were done with the GeneMapper software version 4.1 (Applied Biosystems) according to the manufacturers' instructions. MIRU-VNTR were also compared with SITVIT2, and newly created 24loci MIRU-VNTR International Types (24-MITs) were assigned.

\section{HIV testing}

HIV testing was performed at the Sanitary Unit of enrolment according to the recommendations by the Ministry of Health, Mozambique. Two rapid HIV tests were used sequentially, Unigold Recombinant HIV (Trinity Biotech, Wicklow, Ireland) and Determine HIV-1/2 (Abbot, Tokyo, Japan). Samples were tested first with Determine and reported only when negative. Positive samples were confirmed with Unigold. All tests were performed and interpreted according to the manufacturer's instructions.

\section{Statistical analysis}

Statistical analysis was performed on Data Analysis and Statistical Software (STATA), version 13. Descriptive statistics was used for summarizing demographics data. Categorical variables were presented using frequencies and percentages. Bivariate analyses were performed for TB lymphadenitis versus HIV status, HIV status $x$ lineage using chi-square test. Multinomial Logistic regression model were created with TB lymphadenitis as outcome and sex age and HIV status included as predictors. Interactions were tested and all were not statistically significant. Since no interactions were statistically significant, they are not presented.

\section{Phylogenetic analysis}

Phylogenetic relationships were calculated using MultipleLocus Variable-number tandem repeat Analysis (MLVA) Compare software version 1.03 (Genoscreen and Ridom Bioinformatics). MST were drawn from spoligotyping and 24-loci MIRU-VNTR typing, to better visualize probable relationships and dependencies between isolates. The phylogenetic trees connect each genotype based on degree of changes required to go from one allele to another (the distance numbers are visible on each edge). Solid black line denotes one unique change between two patterns, while solid grey line denotes 2 changes, bold dashed line denotes 3 changes, and thin dotted line represents 4 or more changes. The size of the circle is proportional to the total number of isolates. The colour of the circles indicates the phylogenetic lineage to which the specific pattern belongs. International Types (IT) numbers appear inside nodes. Both SIT and 24-MIT appeared inside nodes of the tree combining spoligotypes and 24-loci MIRU-VNTR.

\section{Additional files}

Additional file 1: Full description of MIRU-VNTR and spoligotyping
analysis in this study. *Newly created 12, 15, or 24-MIRU International
Types (MIT) were followed by an asterisk. Letters x, y, and z represent
respectively MIRU-VNTR double alleles values of loci MIRU26, Mtub21, and
Mtub30 (i.e. 7(+5), 5(+1), and 4(+2)). Letters A, B, C, and D, represent
respectively MIRU-VNTR loci number of copies 10, 11, 12, and 13.
**Mixed infection, Manu 2 isolate. (XLSX 15 kb)
Additional file 2: MST illustrating evolutionary relationships
between the M. tuberculosis spoligotypes of this study ( $\boldsymbol{n}=\mathbf{4 4}$
isolates). Additional file 2 A was drawn using spoligotyping alone,
Additional file 2B was drawn using 24-loci MIRU-VNTR typing alone, and
Additional file 2C was drawn using the combination of both spoligotyping
and 24-loci MIRU-VNTR. (PPTX 402 kb)

\section{Competing interests}

The authors declare that they have no competing interests.

\section{Authors' contributions}

SOV participated in the design and conduction of the study, applied the questionnaires, performed the molecular assays, data analysis and drafting of manuscript; SG participated in the molecular assay, data analysis and critical revision of manuscript, LM applied the questionnaires, performed cultures 
and isolation of mycobacteria, data analysis and critical revision of manuscript; MA participated in sample collection and critical revision of manuscript; FCF participated in sample collection and critical revision of manuscript; EM participated in sample collection and critical revision of manuscript; DC participated in the data analysis and critical revision of manuscript; JMM participated in the statistical data analysis and critical revision of manuscript; NR participated in the data analysis and critical revision of manuscript; MCN participated in the design of the study and critical revision of manuscript; AM participated in the design of the study and critical revision of manuscript; CC participated in the conception and design of the study, supervision of the sample collection process and critical revision of the manuscript; RG participated in the design of the study, general supervision of the research, molecular assay and critical revision of the manuscript; GK participated in the conception and design of the study, general supervision of the research, and critical revision of the manuscript; TK participated in the conception and design of the study, general supervision of the research, and critical revision of the manuscript. All authors read and approved the final version of the manuscript.

\section{Acknowledgements}

This study was funded by the Swedish International Development Cooperation Agency / Department for Research Cooperation (Sida/SAREC) through Eduardo Mondlane University and Karolinska Institutet Research and Training (KIRT) collaboration. We thank the staff of the Pathology Service, at Maputo Central Hospital, who assisted with the sample collection; the nurses who assisted in the data collection and the staff of the National Tuberculosis Reference Laboratory, who assisted in sample processing and culture. We also thank Mikael Mansjo from the Public Health Agency of Sweden, for his support on the molecular analysis.

\section{Author details}

${ }^{1}$ National Institute of Health, Ministry of Health, Maputo, Mozambique. ${ }^{2}$ Faculty of Veterinary, Eduardo Mondlane University, Maputo, Mozambique. ${ }^{3}$ Department of Clinical Science and Education, Karolinska Institutet, Stockholm, Sweden. ${ }^{4}$ Department of Microbiology, Public Health Agency of Sweden, Solna, Sweden. ${ }^{5}$ Pathology Service, Maputo Central Hospital, Ministry of Health, Maputo, Mozambique. ${ }^{6}$ Department of Pathology, Faculty of Medicine, Eduardo Mondlane University, Maputo, Mozambique. 'WHO Supranational TB Reference Laboratory, Tuberculosis \& Mycobacteria Unit, Institut Pasteur de la Guadeloupe, Abymes, Guadeloupe, France. ${ }^{8}$ Life and Health Sciences Research Institute (ICVS), School of Health Sciences, University of Minho, Braga, Portugal. ${ }^{9}$ ICVS/3B's, PT Government Associate Laboratory, Braga/Guimarães, Portugal.

Received: 23 June 2015 Accepted: 12 November 2015

Published online: 21 November 2015

\section{References}

1. World Health Organization. Global tuberculosis report 2014. Geneva: World Health Organization; 2014.

2. INSIDA 2009, Relatório final Inquérito nacional de prevalência, riscos Comportamentais e Informação sobre o HIV e SIDA em Moçambique [Internet]. Moçambique: Ministério da Saúde, Instituto Nacional de Saúde Maputo, Moçambique; Instituto Nacional de Estatística Maputo, Moçambique; ICF Macro Calverton, MD, EUA; 2010 p. 310. Available from: https://dhsprogram.com/pubs/pdf/AlS8/Als8.pdf.

3. Viegas SO, Machado A, Groenheit R, Ghebremichael S, Pennhag A, Gudo PS, et al. Molecular diversity of Mycobacterium tuberculosis isolates from patients with pulmonary tuberculosis in Mozambique. BMC Microbiol. 2010;10:195.

4. Adelina Machado. Mapping of the distribution of Mycobacterium bovis strains involved in bovine tuberculosis in Mozambique, University of Stellembosh. 2015.

5. Tanner M, Inlameia O, Michel A, Maxlhuza G, Pondja A, Fafetine J, et al. Bovine Tuberculosis and Brucellosis in Cattle and African Buffalo in the Limpopo National Park. Mozambique: Transbound Emerg Dis; 2014. Jan;n/a - n/a.

6. Moiane I, Machado A, Santos N, Nhambir A, Inlamea O, Hattendorf J, et al. Prevalence of bovine tuberculosis and risk factor assessment in cattle in rural livestock areas of Govuro District in the Southeast of Mozambique. PLoS One. 2014;9(3), e91527.
7. Müller B, Dürr S, Alonso S, Hattendorf J, Laisse CJM, Parsons SDC, et al. Zoonotic Mycobacterium bovis-induced tuberculosis in humans. Emerg Infect Dis. 2013;19(6):899-908

8. Ayele WY, Neill SD, Zinsstag J, Weiss MG, Pavlik I. Bovine tuberculosis: an old disease but a new threat to Africa. Int J Tuberc Lung Dis Off I Int Union Tuberc Lung Dis. 2004;8(8):924-37.

9. Cosivi O, Grange JM, Daborn CJ, Raviglione MC, Fujikura T, Cousins D, et al. Zoonotic tuberculosis due to Mycobacterium bovis in developing countries. Emerg Infect Dis. 1998;4(1):59-70.

10. Cicero R, Olivera H, Hernández-Solis A, Ramírez-Casanova E, Escobar-Gutiérrez A. Frequency of Mycobacterium bovis as an etiologic agent in extrapulmonary tuberculosis in HIV-positive and -negative Mexican patients. Eur J Clin Microbiol Infect Dis Off Publ Eur Soc Clin Microbiol. 2009;28(5):455-60.

11. Firdessa R, Berg S, Hailu E, Schelling E, Gumi B, Erenso G, et al. Mycobacterial lineages causing pulmonary and extrapulmonary tuberculosis. Ethiopia Emerg Infect Dis. 2013;19(3):460-3.

12. Gumi B, Schelling E, Berg S, Firdessa R, Erenso G, Mekonnen W, et al. Zoonotic transmission of tuberculosis between pastoralists and their livestock in South-East Ethiopia. EcoHealth. 2012;9(2):139-49.

13. Michel AL, Müller B, van Helden PD. Mycobacterium bovis at the animal-human interface: a problem, or not? Vet Microbiol. 2010;140(3-4):371-81.

14. Oloya J, Opuda-Asibo J, Kazwala R, Demelash AB, Skjerve E, Lund A, et al. Mycobacteria causing human cervical lymphadenitis in pastoral communities in the Karamoja region of Uganda. Epidemiol Infect. 2008; 136(5):636-43.

15. Popescu MR, Călin G, Strâmbu I, Olaru M, Bălăşoiu M, Huplea V, et al. Lymph node tuberculosis - an attempt of clinico-morphological study and review of the literature. Romanian J Morphol Embryol Rev Roum Morphol Embryol. 2014;55(2 Suppl):553-67.

16. Baltazar CS, Horth R, Inguane C, Sathane I, César F, Ricardo H, et al. HIV prevalence and risk behaviors among Mozambicans working in south African mines. AIDS Behav. 2015;19(S1):59-67.

17. Bauer J, Andersen ÅB, Kremer K, Miörner H. Usefulness of spoligotyping to discriminate IS6110 Low-copy-number mycobacterium tuberculosis complex strains cultured in Denmark. J Clin Microbiol. 1999;37(8):2602-6.

18. Cronin WA, Golub JE, Magder LS, Baruch NG, Lathan MJ, Mukasa LN, et al. Epidemiologic usefulness of spoligotyping for secondary typing of mycobacterium tuberculosis isolates with Low copy numbers of IS6110. J Clin Microbiol. 2001;39(10):3709-11.

19. Goyal M, Saunders NA, van Embden JD, Young DB, Shaw RJ. Differentiation of Mycobacterium tuberculosis isolates by spoligotyping and IS6110 restriction fragment length polymorphism. J Clin Microbiol. 1997;35(3):647-51.

20. Van Soolingen D. Molecular epidemiology of tuberculosis and other mycobacterial infections: main methodologies and achievements. J Intern Med. 2001;249(1):1-26

21. Bem C, Patil PS, Bharucha H, Namaambo K, Luo N. Importance of human immunodeficiency virus-associated lymphadenopathy and tuberculous lymphadenitis in patients undergoing lymph node biopsy in Zambia. Br J Surg. 1996;83(1):75-8.

22. Bezabih M, Abdissa A, Gadisa E, Aseffa A. Patterns of enlarged cervical lymph nodes among HIV positive and negative patients in southwestern Ethiopia: a cytopathlogic analysis. Ethiop Med J. 2014;52(1):19-25.

23. Sibanda EN, Stanczuk G. Lymph node pathology in Zimbabwe: a review of2194 specimens. QJM Int J Med. 1993;86(12):811-7.

24. Bruchfeld J, Correia-Neves M, Källenius G. Tuberculosis and HIV Coinfection, Cold Spring Harb Perspect Med. 2015. Feb 26.

25. Ronacher K, Joosten SA, van Crevel R, Dockrell HM, Walzl G, Ottenhoff THM. Acquired immunodeficiencies and tuberculosis: focus on HIV/AIDS and diabetes mellitus. Immunol Rev. 2015;264(1):121-37.

26. Saikia UN, Dey P, Jindal B, Saikia B. Fine needle aspiration cytology in lymphadenopathy of HIV-positive cases. Acta Cytol. 2001;45(4):589-92.

27. Sarma PK, Chowhan AK, Agrawal V, Agarwal V. Fine needle aspiration cytology in HIV-related lymphadenopathy: experience at a single centre in north India. Cytopathol Off J Br Soc Clin Cytol. 2010;21 (4):234-9.

28. Shenoy R, Kapadi SN, Pai KP, Kini H, Mallya S, Khadilkar UN, et al. Fine needle aspiration diagnosis in HIV-related lymphadenopathy in Mangalore. India Acta Cytol. 2002;46(1):35-9.

29. Tirumalasetti N, Prema LP. Lymph nodes cytology in HIV seropositive cases with haematological alterations. Indian J Med Res. 2014;139(2):301-7.

30. Biadglegne F, Tesfaye W, Sack U, Rodloff AC. Tuberculous lymphadenitis in northern Ethiopia: in a public health and microbiological perspectives. PLoS 
ONE [Internet]. 2013;8:12. Available from: http://www.ncbi.nlm.nih.gov/pmc/ articles/PMC3857213/.

31. Beyene D, Bergval I, Hailu E, Ashenafi S, Yamuah L, Aseffa A, et al. Identification and genotyping of the etiological agent of tuberculous lymphadenitis in Ethiopia. J Infect Dev Ctries. 2009;3(6):412-9.

32. Srilohasin P, Chaiprasert A, Tokunaga K, Nishida N, Prammananan T, Smittipat N, et al. Genetic diversity and dynamic distribution of mycobacterium tuberculosis isolates causing pulmonary and extrapulmonary tuberculosis in Thailand. J Clin Microbiol. 2014;52(12):4267-74.

33. Rasolofo Razanamparany V, Ménard D, Aurégan G, Gicquel B, Chanteau S. Extrapulmonary and pulmonary tuberculosis in Antananarivo (Madagascar): high clustering rate in female patients. J Clin Microbiol. 2002;40(11):3964-9.

34. Gomes T, Vinhas SA, Reis-Santos B, Palaci M, Peres RL, Aguiar PP. Extrapulmonary tuberculosis: mycobacterium tuberculosis strains and host risk factors in a large urban setting in brazil. PLOS ONE [Internet]. 2013;8: 10. Available from: http://www.ncbi.n/m.nih.gov/pmc/articles/ PMC3788772/.

35. Nicol MP, Sola C, February B, Rastogi N, Steyn L, Wilkinson RJ. Distribution of strain families of mycobacterium tuberculosis causing pulmonary and extrapulmonary disease in hospitalized children in cape town. South Africa Clin Microbiol. 2005:43(11):5779-81.

36. Garedew L, Mihret A, Ameni G. Molecular typing of mycobacteria isolated from extrapulmonary tuberculosis patients at Debre Birhan Referral Hospital, central Ethiopia. Scand J Infect Dis. 2013;45(7):512-8.

37. Narayanan S, Gagneux S, Hari L, Tsolaki AG, Rajasekhar S, Narayanan PR, et al. Genomic interrogation of ancestral Mycobacterium tuberculosis from south India. Infect Genet Evol J Mol Epidemiol Evol Genet Infect Dis. 2008;8(4):474-83.

38. Singh J, Sankar MM, Kumar P, Couvin D, Rastogi N, Singh S, et al. Genetic diversity and drug susceptibility profile of Mycobacterium tuberculosis isolated from different regions of India. J Infect. 2015;29.

39. Faksri K, Drobniewski F, Nikolayevskyy V, Brown T, Prammananan T, Palittapongarnpim $P$, et al. Epidemiological trends and clinical comparisons of Mycobacterium tuberculosis lineages in Thai TB meningitis. Tuberc Edinb Scotl. 2011;91(6):594-600.

40. Yorsangsukkamol J, Chaiprasert A, Prammananan T, Palittapongarnpim P, Limsoontarakul S, Prayoonwiwat N. Molecular analysis of Mycobacterium tuberculosis from tuberculous meningitis patients in Thailand. Tuberc Edinb Scotl. 2009;89(4):304-9.

41. Viegas SO, Machado A, Groenheit R, Ghebremichael S, Pennhag A, Gudo PS, et al. Mycobacterium tuberculosis Beijing genotype is associated with HIV infection in Mozambique. PLoS One. 2013;8(8), e71999.

42. Warren RM, Victor TC, Streicher EM, Richardson M, Beyers N, Gey van Pittius NC, et al. Patients with active tuberculosis often have different strains in the same sputum specimen. Am J Respir Crit Care Med. 2004;169(5):610-4.

43. Zetola NM, Shin SS, Tumedi KA, Moeti K, Ncube R, Nicol M, et al. Mixed mycobacterium tuberculosis complex infections and false-negative results for rifampin resistance by GeneXpert MTB/RIF Are associated with poor clinical outcomes. J Clin Microbiol. 2014;52(7):2422-9.

44. Pang $Y$, Zhou $Y$, Wang S, Song $Y$, Ou $X$, Zhao B, et al. Prevalence and risk factors of mixed Mycobacterium tuberculosis complex infections in China. J Infect. 2015;29.

45. Wang J-Y, Hsu H-L, Yu M-C, Chiang C-Y, Yu F-L, Yu C-J, et al. Mixed infection with Beijing and non-Beijing strains in pulmonary tuberculosis in Taiwan: prevalence, risk factors, and dominant strain. Clin Microbiol Infect Off Publ Eur Soc Clin Microbiol Infect Dis. 2011;17(8):1239-45.

46. Kidane D, Olobo JO, Habte A, Negesse Y, Aseffa A, Abate G, et al. Identification of the causative organism of tuberculous lymphadenitis in ethiopia by PCR. J Clin Microbiol. 2002;40(11):4230-4.

47. Kazwala RR, Daborn CJ, Sharp JM, Kambarage DM, Jiwa SF, Mbembati NA. Isolation of Mycobacterium bovis from human cases of cervical adenitis in Tanzania: a cause for concern? Int J Tuberc Lung Dis Off J Int Union Tuberc Lung Dis. 2001;5(1):87-91.

48. Addo K, Owusu-Darko K, Yeboah-Manu D, Caulley P, Minamikawa M, Bonsu $F$, et al. Mycobacterial species causing pulmonary tuberculosis at the korle bu teaching hospital, accra, ghana. Ghana Med J. 2007:41(2):52-7.

49. Mawak J, Gomwalk N, Bello C, Kandakai-Olukemi Y. Human pulmonary infections with bovine and environment (atypical) mycobacteria in jos. Nigeria Ghana Med J. 2006;40(4):132-6.

50. Macucule B. Study of the prevalence of bovine tuberculosis in Govuro District. [University of Pretoria, South Africa]: Inhambane Province, Mozambique [MSc]; 2008.
51. Kubica GP, Dye WE, Cohn ML, Middlebrook G. Sputum digestion and decontamination with $\mathrm{N}$-acetyl-L-cysteine-sodium hydroxide for culture of mycobacteria. Am Rev Respir Dis. 1963;87:775-9.

52. Vijdea R, Stegger M, Sosnovskaja A, Andersen AB, Thomsen VØ, Bang D. Multidrug-resistant tuberculosis: rapid detection of resistance to rifampin and high or low levels of isoniazid in clinical specimens and isolates. Eur J Clin Microbiol Infect Dis. 2008;27(11):1079-86.

53. Kamerbeek J, Schouls L, Kolk A, van Agterveld M, van Soolingen D, Kuijper S, et al. Simultaneous detection and strain differentiation of Mycobacterium tuberculosis for diagnosis and epidemiology. J Clin Microbiol. 1997;35(4):907-14.

54. Demay C, Liens B, Burguière T, Hill V, Couvin D, Millet J, et al. SITVITWEB-a publicly available international multimarker database for studying Mycobacterium tuberculosis genetic diversity and molecular epidemiology. Infect Genet Evol J Mol Epidemiol Evol Genet Infect Dis. 2012;12(4):755-66.

55. Supply P, Allix C, Lesjean S, Cardoso-Oelemann M, Rüsch-Gerdes S, Willery E, et al. Proposal for standardization of optimized mycobacterial interspersed repetitive unit-variable-number tandem repeat typing of mycobacterium tuberculosis. J Clin Microbiol. 2006;44(12):4498-510.

\section{Submit your next manuscript to BioMed Central and take full advantage of:}

- Convenient online submission

- Thorough peer review

- No space constraints or color figure charges

- Immediate publication on acceptance

- Inclusion in PubMed, CAS, Scopus and Google Scholar

- Research which is freely available for redistribution 